\title{
A Review of Compressive Sensing Detection for Spatial Modulation in Massive MIMO System
}

Manpreet Kaur and Navjot Kaur

\author{
School of Electronics \& Electrical Engineering, Lovely Professional University, Phagwara - 144402, \\ Jalandhar, Punjab, India; manpreetmehmi94@gmail.com, navjot.12695@lpu.co.in
}

\begin{abstract}
Multiple Input Multiple Output (MIMO) low complexity receiver which utilizes the compressive sensing detection for Spatial Modulation in large scale MIMO system in order to reduce the system complexity. In conventional MIMO system; huge amount of antennas is used at both ends to exploit the multipath propagation. This system maximizes the throughput performance and data rates are increased but only at the cost of high hardware complexity and increased power-consumption. Spatial Modulation Matching Pursuit (SMMP) is the proposed enhanced CS technique used for the improvement of detection performance. Hence, this paper reviews recent research findings concerning normalized Compressive Sensing (CS) detection algorithm, used for Spatial Modulation (SM) in massive MIMO, to lowers the signal processing complexity, which in result improves the energy efficiency of system against that of conventional MIMO system. This strategy is achieved by involving additional structures and sparsity in which a single transmitter antenna or a subset of it is turned on at each case to transmit a certain data. The subset of antenna which is turned on for transmission depends on approaching data bits. Therefore, the total increase in the spectral efficiency of the system is given as base-two logarithm of whole antennas at the transmitter. It reduces the signal processing load at base station and doesn't depend upon any synchronization between transmitters. The Spatial Modulation Matching Pursuit used prevents the Inter Channel Interference (ICI) of the system which in result improves the Bit Error Rate (BER) performance than the typical MIMO system.
\end{abstract}

Keywords: Compressive Sensing, Energy Efficiency, Large-Scale MIMO, Spatial Modulation

\section{Introduction}

MIMO comprises of huge amount of antennas at both the transmitter and receiver $\operatorname{side}^{\frac{1-2}{2}}$. It delivers more data streams in less time, i.e. parallel data. It consists of 3 features based on application areas - Spatial Diversity, Spatial Multiplexing and Beam forming. Spatial Diversity includes the transmission of number of copies of data streams at the transmitter side and the extraction of desired signal at the receiver side. It improves the transmission error and increases the system quality. Spatial Multiplexing includes the transmission of multiple, different data streams from the transmitter and reception from receiver after experiencing the same channel quality as like the single-input-single-output system. It provides the high data rates and increases the system capacity.
Beam forming includes the transmission and reception of directional signal, in sensor arrays. It can be used in order to achieve spatial selectivity ${ }^{2}$. Particularly, the data rates in wireless communication are increased exponentially whereas the total energy consumption is also increased. This happens because of designing large number of antennas at base stations, transmission power and complexity in their signal processing algorithms. There occurs the reduction in the energy efficiency. Therefore, to satisfy the need of efficiency of energy of future wireless communication, technologies like massive MIMO and Spatial Modulation (SM) have been established.

The goal of this paper is to give a review of significant findings related to compressive sensing detection for massive MIMO system. Compressive Sensing detection can be defined as acquiring a signal from series of sampling 
measurement, done with sampling theorem. According to this theorem, the sampling frequency must not be less than the twice of maximum frequency, $f_{s} \geq 2 f_{m}$. Massive MIMO technologies include compressive sensing detection for spatial modulation in massive MIMO. It increases the Energy Efficiency (EE) by incorporating the multiple numbers of antennas at the base stations $s^{3-7}$. The typical linear detection and pre-coding techniques become best in large scale. Unlike conventional MIMO transmission, the additional structure is incorporated for this purpose in which a single transmitter antenna or a subset of them is activated for transmission instead of activating all antennas simultaneously. This strategy reduces the RF complexity and removes the inter-channel interference but also decreases the maximum achievable rates $\frac{8-10}{}$. It requires no synchronization between transmitters.

\section{System Model}

The model used in related findings is the Multiple Access Channel (MAC) of massive MIMO system. It consists of $\mathrm{k}$ number of mobile stations that have $n_{t}$ transmit antennas and an individual base station that have $\mathrm{N}$ receive antennas. $\mathrm{M}$ is the representation for the whole antennas at mobile station. It is given by $M=K \times n_{t}$. The system of multiple access is given by:

$$
Y=H x+w
$$

Where $y \in \mathbf{C}^{N \times 1}$ is the output data at base station and $\boldsymbol{x} \in \mathbf{C}^{M \times 1}$ is the input data by mobile station. $H \in \mathbf{C}^{N \times M} \sim C \mathrm{~N}\left(0, I_{N} \otimes I_{M}\right)$ is a matrix with $\mathrm{h}_{\mathrm{m}, \mathrm{n}}$ as the $\mathrm{m}, \mathrm{n}^{\text {th }}$ complex coefficients. These represent the channel gain of frequency flat fading between $\mathrm{n}^{\text {th }}$ transmitter and $\mathrm{m}^{\text {th }}$ receiver. $\boldsymbol{w} \in \mathbf{C}^{N \times M} \sim C \mathbf{N}\left(0, \sigma_{n}^{2} \boldsymbol{I}_{N}\right)$ denotes the Additive White Gaussian Noise (AWGN) vector with variance $\sigma_{n}^{2} . I_{N}$ and $I_{M}$ are the identity matrices with $\operatorname{order} N \times N$ and $M \times M$ respectively. Also, $\otimes$ represents the Kronecker product ${ }^{\underline{11}}$. The average SNR of MAC $\rho$ is given by

$$
\rho=\frac{\mathbb{E}\left[x^{H} x\right]}{\sigma_{n}^{2}}=\frac{S \cdot \mathbb{E}\left[E_{s}\right]}{\sigma_{n}^{2}}
$$

where $(\cdot)^{H}$ denotes Hermitian transpose, $E_{s}$ refers to symbol energy, $\mathbb{E}$ denotes expected value and $S \leq M$ are the whole antennas that are turned on simultaneously amongst the mobile stations.

\section{Multiple Access Spatial Modulation}

Because of limited number of active antennas used, generalized SM reduces the hardware complexity of multiple antennas devices and conveys extra information onto their spatial position. In SM, the given antennas $n_{a}$ are activated according to the input sequence and the same constellation symbol is transmitted by the transmitter ${ }^{12}$. Without any loss, we supposed that number of antennas activated by users is same: $S=n_{a} \times K$. The transmit signal $\mathbf{x}_{u} \in \mathbf{C}^{n_{\mathrm{t}} \times 1}$ can be expressed as:

$$
\boldsymbol{x}_{u}=\left[0^{\cdots} s_{\downarrow}\left(l_{1} 1\right)^{\dagger} q \cdots s_{1}\left(l_{1} k\right)^{\uparrow} q \cdots 0\right]^{\dagger} T
$$

Where $l_{k} \in\left[1, n_{t}\right]$ represents the active antenna index and $S^{q}$ represents $q^{\text {th }}$ symbol of transmit constellation $^{\mathcal{B}}$.

At the transmitter side, the antennas used per user are the power of 2 and is given by $r=2^{b}$

where $b \triangleq\left[\log _{2}\left(\begin{array}{l}n_{t} \\ n_{a}\end{array}\right)\right\rfloor$

where $\left.\log _{2}\left(\begin{array}{l}n_{t} \\ n_{a}\end{array}\right)\right]$ is antenna indices ${ }^{12}$. It defines the cardinality of $\mathcal{A}$ where it is the set of possible antenna groups. Unlike the typical technologies of MIMO system, SM reduces the interference in the channel and the power consumed of mobile stations, but only at the loss of reduction in gained data rates. A conventional MIMO transmitter transmits $B_{\text {MIMO }}=n_{t} \cdot \log _{2}(Q)$ bits whereas an individual SM transmits $B_{S M}=b+\log _{2}(Q)$ bits in every channel use.

\section{Massive MIMO and Low Complexity Detection}

Massive MIMO includes so many antennas at the base station that increases the complexity and power consumption of the system. For $N \gg M$,one of the result states that the output signal after linear detection fulfills: 


$$
g=\underset{N \rightarrow \infty, M=\text { const. }}{D(H x+w)(\text { a.s. })} \text {. }
$$

Where $\mathrm{D}^{\in} \mathbf{C}^{M \times N}$ is a matrix for the linear detection matrix of the three detectors; matched filter, zero forcing, minimum mean square error and it is given by ${ }^{13}$ :

$$
D^{M F}=H^{H}
$$

$$
D^{M M S E}=\left(H^{H} H+\varsigma I\right)^{-1} H^{H}
$$

Where ${ }^{\varsigma}=\mathrm{M} / \rho \frac{13}{},(\cdot)^{\dagger}(-1)$ is the inverse matrix and ( $\cdot)^{\dagger} \mathbf{I}$ is the pseudo-inverse operation. The importance to increase the large amount of antennas at the transmitter side while using SM degrades the performance of MF, ZF and MMSE detectors. Therefore, a low-complexity detector is proposed in recent research findings in which Compressive Sensing (CS) technology takes the advantage of large-scale MIMO benefits and reduces the detection complexity of hardware. i.e. $N \gg M$ necessarily holds good for massive MIMO.

\section{CS Based Algorithms for SM Detection}

The main problem of typical detectors is that even if only S columns are used for acquiring the information of amplitude and phase signal, but still the complete channel matrix $\mathbf{H}$ must be required for detection. The signal transmitted by $\mathrm{SM}$ contains $S \ll M$ valuable entries which are equivalent to the simultaneously active antenna $S$ and they are defined as $S$-sparse ${ }^{14}$. This improves the signal estimation from compressive techniques. The compressive analysis $\boldsymbol{y} \in \mathbf{R}^{N \times 1}$ of a sparse signal $\boldsymbol{x}$ is given by $\frac{15-17}{}$.

$$
y=\varphi x+e
$$

Where $\boldsymbol{x} \in \mathbf{R}^{M \times 1}$ represents the sparse signal, $e \in \mathbf{R}^{N \times 1}$ represents the measurement error term and $\varphi \in \mathbf{R}^{N \times M}$ represents the measurement matrix. These recent research findings include the performance improvement of the typical MIMO detectors with the exploitation of similarity between (1) and (8). In compressive sensing detection, RIP is the Restricted Isometry Property. It defines whether the recovery of signal is achieved or not for any channel $\varphi=\mathbf{H}^{\frac{15-16}{1}}$. For a channel matrix $\mathbf{H}$ of the MIMO channel, the RIP of order $S$ is satisfied if, for any $S$-sparse signal $\boldsymbol{x}$, the relationships:

$$
\left(1-\delta_{s}\right)\|x\|_{2}^{2} \leq\|H x\|_{2}^{2} \leq\left(1+\delta_{s}\right)\|x\|_{2}^{2}
$$

hold for a constant $\delta_{s} \in(0,1)$. to establish an estimate in the compressive sensing, the detection of the SM signals depends upon the sparsity of SM. For $\delta_{2 s}<\sqrt{2}-1 \frac{17}{2}$, (4) is solved in low-complexity manner and is expressed as:

$$
\begin{aligned}
& \text { subject to }\|H x-y \square\| \square \downarrow 2 \leq \mu \\
& \text { minimise }\|x\|_{1}
\end{aligned}
$$$$
\text { subject to }\|H x-y \square\| \square \downarrow 2 \leq \mu \text { Where }
$$

is a constant that restricts the power of noise with $\|\mathbf{w}\|_{\mathbf{2}} \leq \mu$. We can solve these optimization problems with some convex approaches, which are often highly complex, with high speed techniques. Therefore, they offer a trade-off between performance and complexity. Amongst the variety of CS greedy algorithms, the most efficient scheme named Compressive Sampling Matching Pursuit (CoSaMP) is selected to approximate the solution of $(10)^{18}$. The CoSaMP is a low-complexity algorithm that recovers the indices of active antenna as well as the information of amplitude and phase information of the transmitted signals through a repetitive reconstruction process. It provides the guarantee for the error free detection of sparse signals. The results of this research show that the high amount of antennas for SM detection at the base station exploits the use of this algorithm by performing an analysis of complexity as opposed to ${ }^{18}$. The following approach is considered to improve the detection performance.

\section{CS Detection Technique}

Spatial Modulation Matching Pursuit (SMMP) is the proposed enhanced CS technique used for the improvement of detection performance. In typical CS algorithms, no any prior information regarding the sparse signal is assumed. Only, amount of non-zero entries is considered. When SMMP uses this algorithm, it generates the detection output without any physical sense. Unlike the conventional SM modulation, the detected signal could have more than one active antenna per user. The noise and the inter-channel interference effects raised in the MAC are the causes for this unwanted condition. Therefore, in the transmitted signal, the extra prior information about the distribution of the non-zero entries is incorporated to reduce these effects and to further improves the performance ${ }^{19}$. The detection algorithm of SMMP explicitly indicates that it corresponds to a particularization to SM operation of the 
structured CoSaMP iteration developed in ${ }^{20}$. Particularly, the errors are reduced in the active antennas' identification by SMMP with the exploitation of the known distribution of the non-zero entries $\frac{19-20}{}$. The requirement of an epoch to determine the amount of antennas that are turned on is beneficial to meet the converging point of the algorithm. It improves the speed of algorithm. With the estimation of the largest components of the transmitted signal, the algorithm identifies the antennas that are turned on. The residual signal $r \in \mathbf{C}^{N \times 1}$ employed by the algorithm is expressed as:

$$
r \triangleq y-H x^{i}=H\left(x-x^{i}\right)+w
$$

Where $\tilde{\mathbf{x}}^{i} \in \mathbf{C}^{M \times 1}$ is the approximate transmitted signal at the $i^{\text {th }}$ iteration $\frac{21-22}{}$. The decision matrix determines the apparently reasonable active antennas $\mathbf{c} \in \mathbf{C}^{M \times 1}$ and can be expressed as

$$
c=H^{H} r \text { (12) }
$$

With the help of this decision matrix, a set of decision variables $\boldsymbol{\Omega}$ is formed by the active antenna estimation process. An estimate of the apparently reasonable active antennas is provided by the set $\boldsymbol{\Omega}$. The LS approximation reduces the system complexity and improves the performance with respect to the conventional linear alternatives that depend on its efficiency. This implement a ZF detector in which only previously included columns in the support are inverted. This exploits the benefits of large-scale MIMO detection and reduces the complexity. Several parameters can be modified to adjust the trade-off between complexity and performance and they are:

- Maximum epoch of SMMP: The whole iterations denoted by $\mathbb{Z}\left(i \rrbracket_{\max }\right)_{\text {adjust the performance of }}$ system and concludes the complexity and detection accuracy of the algorithm.

- Output entries of the matched filter: The output entries of the matched filter (MF) denoted by $(k)$ determines the dimensions of the LS problem and affects the complexity and performance.

- Maximum epoch of the iterative LS: The maximum epoch of the iterative LS is denoted by $i_{\downarrow} \max ^{\uparrow} l s$. While using iterative algorithms, every epoch improves the solution accuracy ${ }^{23}$. Therefore, a complexity and performance trade-off occurs. On the basis of need of communication, it can be optimized at the BS.
In the proposed approach, the communication systems with $N \geq M$ are also considered which are not taken in the conventional CS. SMMP reduces the typical detectors' complexity as well as provides improvement in performance.

\section{Complexity Analysis of Least Squares Problem}

The order of complexity performs the computational complexity analysis. It was applied in $\frac{14,18}{}$ to evaluate the complexity of algorithm. Because of repetitive nature of the greedy CS algorithms, the order of complexity provides the inaccurate performance of the total operations. Therefore, a more precise approach is adopted in the recent research findings. On contrary to the results obtained $\mathrm{in}^{\frac{14,18}{18}}$ it is concluded in recent findings that the LS problem can control the whole complexity. Computational hardware complexity can influence the whole floating point operations (flops) ${ }^{18}$. Therefore, the requirement of LS algorithm efficient implementation is to decrease the complexity of the used method. Generally, two methods named direct and iterative procedures are used to solve the least square problem ${ }^{23}$.

- Direct Method: Direct Method includes the QR and the Cholesky decompositions. They depend upon creating the equations. These equations can be solved without any difficulty ${ }^{23}$. This method requires the storage intensive decompositions.

- Iterative Method: Based on the instantaneous residual, the LS problem is solved by improving starting solution in the repetitive $\operatorname{method}^{24}$. These accesses avoid the high storage decompositions.

The operations used QR, CG etc. analyse the complexity ${ }^{24}$. CS-based algorithm depends on the increment in the accuracy as the algorithm involved $\mathrm{in}^{24}$. In results, it improves the speed of convergence. The difference between direct LS method is that Cholesky decomposition is less complex than QR decomposition even though it is inaccurate. Therefore, due to the high complexity, the QR decomposition is not preferred in the LS problem. The improvement in the complexity of the iterative methods does not reduce the operations used as the total complexity and epoch are inversely proportional. 


\section{Analysis of Energy Efficiency}

As we know that the study of EE is very important to increase the battery life-time. Therefore, in the recent findings, the EE model is defined to characterise the EE improvements. The EE is expressed as the rate per milliwatt of total consumed power by using the metric $\frac{25,27-32}{}$

$$
E=\frac{S_{s}}{\sum_{u=1}^{k} P_{u}} \text { subject to } B E R \leq B E R_{o b j}
$$

Where $\mathbf{B E R}_{o b j}$ is the objective average Bit Error Rate (BER), $S_{e}$ is the spectral efficiency calculated in (bpcu) and $P_{u}$ is the whole power consumption (mW). It is required to gain a given $\mathbf{B E R}_{o b j}$. The total power consumption per mobile station is given by:

$$
P_{u}=P_{C u}+P_{T u}=\left[P_{\psi}+P_{\psi}\right]\left[\left(\sum_{j=1}^{n_{t}} p_{u, j}\right) \cdot \zeta\right]
$$

Where $P_{C u}=\left[P_{\psi}+P_{\psi}\right]_{\text {denotes the whole power con- }}$ sumption not including the power amplifier (PA) and it is classified into two factors: $P_{\psi}$ and $P_{\psi} . P_{\psi}$ represents the static power consumption and $P_{\psi}$ represents the circuit power consumption.

$$
P_{T u}=\left[\left(\sum_{j=1}^{n_{t}} p_{u, j}\right) \cdot \zeta\right] \text { is the power consumption of power }
$$
amplifier. It depends on the parameter $\zeta$ which is equivalent to $\frac{v}{\eta}$. It also depends upon the requisite signal power, transmitted by all antennas individually $p_{u, j}$. The factor $i$ is the peak-to-average power ratio (PAPR) which reduces the unwanted distortion in the signal of system and it depends on the modulation ${ }^{26}$. Ç represents the efficiency of power amplifier ${ }^{27}$. The whole EE can be given by:

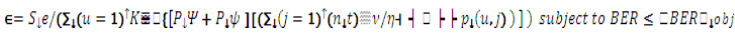

Here, the efficiency factor is equivalent to $\eta=0.35$ and it is a class-A power amplifier ${ }^{27}$. Also the potential future research can be carried out in which the BER performance for this CS scheme can be characterized analytically in the large-scale.

\section{Conclusion}

This paper gives a review of related research findings related to the operation of CS based algorithm for SM and illustrates that how the compressive sensing detection techniques can reduce the system complexity and improves the system performance by incorporating the additional structure and sparsity of transmitted signals in the MAC. It represents an alternative of low-complexity to increase the EE in the MAC. It also defines the ways of maximizing the benefits of this scheme when high amount receive antennas at the BS are used due to its faster convergence and improved performance. The paper has highlighted possible future research directions within this field.

\section{References}

1. Li G, et al. Energy-Efficient Wireless Communications: Tutorial, Survey, and Open Issues, IEEE Wireless Communication. 2011 Dec; 18(6):28-35.

2. Bjornson E, Sanguinetti L, Hoydis J, Debbah M. Optimal Design of Energy-Efficient Multi-User MIMO Systems: Is Massive MIMO the Answer? IEEE Trans. Wireless Communication. 2015 Jun; 14(6):3059-75.

3. Di Renzo M, Haas H, Ghrayeb A. Sugiura S, Hanzo L. Spatial Modulation for Generalized MIMO: Challenges, Opportunities, and Implementation, Proc. IEEE. 2014 Jan; 102(1):56-103.

4. Rusek F, et al. Scaling up MIMO: Opportunities and Challenges with Very Large Arrays, IEEE Signal Process. Mag. 2013 Jan; 30(1):40-60.

5. Hoydis J, Ten Brink S, Debbah M. Massive MIMO in the UL/DL of Cellular Networks: How Many Antennas do we Need? IEEE J. Sel. Areas Communication. 2013 Feb; 31(2):160-71.

6. Masouros C, Sellathurai M, Ratnarajah T. Large-Scale MIMO Transmitters in Fixed Physical Spaces: The Effect of Transmit Correlation and Mutual Coupling, IEEE Trans. Communication. 2013 Jul; 61(7):2794-804.

7. Masouros C, Matthaiou M. Space-Constrained Massive MIMO: Hitting the Wall of Favourable Propagation, IEEE Communication, Letters. 2015 May; 19(5):771-74.

8. Ha D, Lee K, Kang J. Energy Efficiency Analysis with Circuit Power Consumption in Massive MIMO Systems, In: Proc. IEEE 24th Int. Symp. PIMRC, September 2013.

9. Yang P, Di Renzo M, Xiao Y, Li S, Hanzo L. Design Guidelines for Spatial Modulation, IEEE Communication Surveys Tuts., 1st Quart. 2015; 17(1):6-26.

10. Di Renzo M, Haas H, Grant PM. Spatial Modulation for Multiple Antenna Wireless Systems: A Survey, Ieee Communication Mag. 2011 Dec; 49(12);182-91.

11. Golub GH, Van Loan CF. Matrix Computations, Baltimore, MD, USA: The Johns Hopkins Univ. Press, 2012, 3.

12. Younis A, Serafimovski N, Mesleh R, Haas H. Generalised Spatial Modulation, In: Proc. Conf. Rec. 44th ASILOMAR, 2010 Nov, p. 1498-502. 
13. Peel C, Hochwald B, Swindlehurst A. A VectorPerturbation Technique for Near-Capacity Multi-Antenna Multiuser Communication-Part I: Channel Inversion and Regularization, IEEE Trans. Communication. 2005 Jan; 53(1):195-202.

14. Yu C-M, et al. Compressed Sensing Detector Design for Space Shift Keying in MIMO Systems, IEEE Communication Lett. 2012 Oct; 16(10);1556-59.

15. Candès EJ, Wakin MB. An Introduction to Compressive Sampling, IEEE Signal Process. Mag. 2008 Mar; 25(2):2130.

16. Candès E. Compressive Sampling, In: Proc. Int Congr. Math., Madrid: Spain, 22-30 August 2006, p. 1433-52.

17. Candes E, Romberg J; Tao T. Stable Signal Recovery from Incomplete and Inaccurate Measurements, Commun. Pure Appl. Math. 2006 Aug; 59(8):1207-23.

18. Needell D, Tropp JA. CoSaMP: Iterative Signal Recovery from Incomplete and Inaccurate Samples, Appl. Comput. Harmonic Anal. 2009 May; 26(3):301-21.

19. Eldar YC, Mishali M. Robust Recovery of Signals from a Structured Union of Subspaces, IEEE Trans. Inf. Theory. 2009 Nov; 55(11):5302-16.

20. Baraniuk RG, Cevher V, Duarte MF, Hegde C. Model Based Compressive Sensing, IEEE Trans. Inf. Theory. 2010 Apr; 56(4):1982-2001.

21. Zheng J. Low-Complexity Detector for Spatial Modulation Multiple Access Channels with a Large Number of Receive Antennas, IEEE Communication Lett. 2014 Nov; 18(11):2055-58.

22. Rao X, Lau VK. Distributed Compressive CSIT Estimation and Feedback for FDD Multi-User Massive MIMO Systems, IEEE Trans. Signal Process. 2014 Jun; 62(12):3261-71.

23. Vandenberghe L. Applied Numerical Computing, Univ. Calif., Los Angeles, CA, USA: Univ. Lecture, 2011.

24. Björck A. Numerical Methods for Least Squares Problems, Philadelphia, PA, USA: SIAM, 1996.
25. Wang S, Li Y, Zhao M, Wang J. Energy Efficient and Low-Complexity Uplink Transceiver for Massive Spatial Modulation MIMO, IEEE Trans. Veh. Technol. 2015; 64(10):4617-32.

26. Kmutha D, Amutha Prabha N. Performance Analysis of PAPR Reduction in LTE System, Indian Journal of Science and Technology, 2016 Aug; 9.

27. Cui S, Goldsmith AJ, Bahai A. Energy-Constrained Modulation Optimization, IEEE Trans. Wireless Communication. 2005 Sep; 4(5):2349-60.

28. Miao G. Energy-Efficient Uplink Multi-User MIMO, IEEE Trans. Wireless Communication. 2013 May; 12(5):2302-13.

29. Ntontin K, Di Renzo M, Perez-Neira A, Verikoukis C. Towards the Performance and Energy Efficiency Comparison of Spatial Modulation with Conventional Single-Antenna Transmission Over Generalized Fading Channels, In: Proc. IEEE Int. Workshop CAMAD, 2012 Sep, p. 120-24.

30. Masouros C, Sellathurai M, Ratnarajah T. Maximizing Energy Efficiency in the Vector Precoded MU-MISO Downlink by Selective Perturbation, IEEE Trans. Wireless Communication. 2014 Sep; 13(9):4974-84.

31. Masouros C, Sellathurai M, Ratnarajah T. Vector Perturbation Based on Symbol Scaling for Limited Feedback MISO Downlinks, Ieeetrans. Signal Process, 2014 Feb; 62(3):562-71.

32. Garcia-Rodriguez A, Masouros C. Power-Efficient Tomlinson-Harashima Precoding for the Downlink of Multi-User MISO Systems, IEEE Trans. Commun. 2014 Jun; 62(6):1884-96.

33. Garcia-Rodriguez A, Masouros C. Power Loss Reduction for MMSETHP with Multi-Dimensional Symbol Scaling, IEEE Communication, Lett. 2014 Jul; 18(7):1147-50. 